\title{
Idade de Desmame de Cordeiros Deslanados para Terminação em Confinamento, no Litoral Norte da Bahia ${ }^{1}$
}

\section{Davi Correia de Freitas ${ }^{2}$, Gabriel Jorge Carneiro de Oliveira ${ }^{3}$, Soraya Maria Palma Jaeger ${ }^{3}$, Adriana da Silva Rodrigues Cavalcanti ${ }^{4}$, Carlos Alberto da Silva Ledo ${ }^{5}$, Paulo Emílio Landulfo Medrado de Vinhaes Torres ${ }^{2}$, Antônio de Oliveira Leite Filho², Paulo Fontes de Amorim e Santana², Danilo Câmara de Almeida²}

\begin{abstract}
RESUMO - Trinta e dois cordeiros (16 machos e 16 fêmeas) deslanados mestiços de Santa Inês, criados com as matrizes em pastagem de Brachiaria humidicola e suplementados em creep-feeding no período noturno, foram distribuídos em delineamento inteiramente casualizado, em esquema fatorial 4x2 - quatro idades de desmame (56, 70, 84 e 98 dias) e sexo, com o objetivo de determinar a melhor idade para o desmame desses animais. Ao desmame, os cordeiros foram confinados, até completarem 126 dias de vida, em baias individuais, onde receberam feno de Tifton 85 (Cynodon spp. cv Tifton 85), água e sal mineral ad libitum e ração concentrada na proporção de $2 \%$ do peso vivo. Houve efeito significativo da interação dos fatores idade de desmame e sexo sobre GPC e GPD, em que os cordeiros desmamados aos 84 dias foram superiores às cordeiras desmamadas com a mesma idade. Não se observou efeito significativo na interação dos fatores idade de desmame e sexo sobre GPND e CAC. Verificou-se efeito quadrático da idade de desmame para as variáveis GPND, GPD e CAC. Não se observou efeito significativo do sexo em nenhum dos parâmetros avaliados. A idade de desmame de 76 dias proporcionou maximização dos parâmetros GPND e GPD, enquanto a CAC mais eficiente foi observada nos animais desmamados aos 72 dias.
\end{abstract}

Palavras-chave: alimentador restrito, Brachiaria, conversão alimentar, ovino

\section{Weaning Age on Fedlot Hair Sheep Lambs Performance in the Northern Litoral of Bahia}

ABSTRACT - Thirty-two crossbred Santa Inês hair sheep lambs (16 males and 16 females) bred with their female progenitors on pasture of Brachiaria humidicola grass, supplemented in creep-feeding during the night, were allotted to a completely randomized design, in a factorial arrangement $4 \times 2$, four weaning ages (56, 70, 84 and 98 days) and sex to determine the best weaning age. At weaning, the lambs were confined in individual pens until 126 days old, when were daily fed Tifton 85 hay, water and mineral salt ad libitum and concentrate diet in the proportion of $2 \%$ body weight. There was a significant effect of weaning age vs sex interaction on DWC and DWG, where the males weaned at 84 days old were heavier than the females. No significant interaction of weaning age vs sex on DWBW and CFC was detected. Quadratic effects of weaning age on DWBW, DWG and CFC were noticed. No significant effect of sex on any of the studied parameters was detected. Seventy-six days weaned lambs maximized DWBW and DWG, while the most effective CFC was observed in seventy-two days weaned animals.

Key Words: Brachiaria, creep-feeding, feed:gain ratio, sheep

\section{Introdução}

O litoral norte da Bahia apresenta fatores climáticos favoráveis à produção de forragens tropicais altos índices de luminosidade, temperaturas médias em torno de $25^{\circ} \mathrm{C}$, baixa amplitude térmica anual e precipitação média de $1.500 \mathrm{~mm} / \mathrm{ano}$. Entretanto, Siqueira (2000a) alerta que essas condições climáticas são favoráveis à proliferação helmíntica nas pastagens.

Segundo Boas (1990), os ovinos se infectam por helmintos ao ingerirem forragem contaminada. Os cordeiros compõem a categoria animal mais sensível à verminose, que provoca redução na absorção de nutrientes, na digestão e no consumo voluntário dos alimentos (Siqueira, 2000), além de redução da taxa de crescimento (Barros et al., 1996) e aumento da taxa de mortalidade (Guimarães Filho et al., 2000) justamente dentro do período definido por Oliveira (2002), de até 90 dias de idade, como o de maior capacidade produtiva dos ovinos.

Siqueira (2000b) afirma que a ovelha produz $75 \%$ do total da lactação até os 56 dias pós-parto. Silva Sobrinho (2001) relata que, a partir dos 56 dias, o cordeiro já tem a capacidade de um ovino adulto para 
digerir alimento sólido, estando apto ao desmame. Contudo, Oliveira et al. (1996) recomendam o desmame dos 70 aos 84 dias de idade e Castillo et al. (1973), dos 75 a 90 dias.

Visando reduzir o tempo de exposição dos cordeiros aos helmintos nas pastagens com altas cargas parasitárias e de baixo valor nutritivo, Siqueira (2000a) sugere a antecipação do desmame, seguida da terminação em confinamento. Para que os ovinos sejam desmamados mais cedo, o autor recomenda a suplementação exclusiva dos lactentes por meio do uso de alimentador privativo desde os dez dias de idade. De acordo com Neres et al. (2001), esta prática eleva o ganho de peso, além de melhorar a resposta imunitária dos cordeiros frente às infecções verminóticas (Haile et al., 2002).

De acordo com Nudell (2001), para determinação da idade ótima de desmame de cordeiros para terminação em confinamento, é necessária a realização de estudos de parâmetros produtivos, de forma a explorar a máxima eficiência nas fases de cria e terminação.

Objetivou-se, com este trabalho, determinar a melhor idade para o desmame de cordeiros deslanados, mestiços de Santa Inês, para terminação em confinamento no litoral norte da Bahia. Foram analisados os parâmetros ganho de peso diário do nascimento ao desmame (GPND), ganho de peso diário em confinamento (GPC), ganho de peso diário do nascimento ao abate (GPD) e conversão alimentar do concentrado no confinamento (CAC), entendendo-se por CAC a relação entre $\mathrm{kg}$ de ganho de peso por $\mathrm{kg}$ de concentrado consumido.

\section{Material e Métodos}

O experimento foi conduzido na Fazenda Lagoa, localizada no município de Jandaíra, litoral norte da Bahia, situado a $11^{\circ} 33^{\prime} 45^{\prime \prime}$ de latitude sul e $37^{\circ} 48^{\prime} 45^{\prime \prime}$ de longitude oeste, com altitude média de 146 metros. O clima na região é do tipo quente e úmido, com temperatura média anual de $24,8^{\circ} \mathrm{C}$ e precipitação de $1.500 \mathrm{~mm}$. O solo é caracterizado por baixa fertilidade natural, com $\mathrm{pH}$ de 5,38 e baixos níveis de fósforo $(0,8 \mathrm{ppm})$.
Foram utilizados 32 cordeiros (16 machos e 16 fêmeas), filhos de reprodutores Santa Inês puros de origem (PO) e matrizes deslanadas sem raça definida (SRD) com ordem de parto entre três e cinco, nascidos de partos simples entre $18 / 10$ e 04/11/2002, com pesos vivos médios ao nascimento de $3,85 \mathrm{~kg}$ (de 3,0 a 4,6 kg), ao desmame de $13,53 \mathrm{~kg}$ e ao abate, de $20,45 \mathrm{~kg}$. O período experimental teve duração de seis meses.

Os cordeiros foram manejados com as ovelhas progenitoras em 14 piquetes de 1,5 ou 2,0 hectares, formados com pastagem de Brachiaria humidicola, com lotação animal média de cinco matrizes/ha, período de ocupação de 1,5 ou 2 dias (proporcional à área de cada piquete) e intervalo de pastejos de 24 dias. Diariamente, às $17 \mathrm{~h}$, os cordeiros eram recolhidos num galpão, onde tinham acesso ao alimentador privativo (creep-feeding) até às $7 \mathrm{~h}$ do dia seguinte, quando retornavam aos piquetes. A ração do alimentador privativo foi composta por $68 \%$ de milho moído, $29 \%$ de farelo de soja e 3\% de sal mineral $(19,37 \%$ de PB e $75,57 \%$ de NDT), verificando-se consumo diário médio de $125 \mathrm{~g}$.

Apesar de terem recebido vermífugo homeopático constantemente, misturado ao sal mineral, todos os animais do experimento foram tratados, no dia do desmame, com medicamento à base de Moxidectina ${ }^{1}$ a $1 \%$, na dosagem de $1 \mathrm{~mL} / 50 \mathrm{~kg}$ de PV.

Após o desmame, os cordeiros foram confinados até os 126 dias em baias individuais de piso cimentado, com área útil de $1,5 \mathrm{~m}^{2}$ e cama de areia, onde receberam feno de Cynodon spp. cv Tifton 85 (92,45\% de MS, 9,73\% de PB, 78,85\% de FDN, 39,05\% de FDA e 1,81\% de EE), água e sal mineral à vontade. A ração concentrada $(19,95 \%$ de PB e $75,46 \%$ de NDT), composta de $66,4 \%$ de milho em grão, $30,6 \%$ farelo de soja e 3,0\% premix vitamínico mineral, foi fornecida na proporção de $2 \%$ do $\mathrm{PV}$ por dia, dividida em duas refeições diárias, às 8 e $18 \mathrm{~h}$.

O cálculo dos níveis de PB e NDT das rações concentradas do alimentador privativo e do confinamento se baseou nas tabelas de composição nutricional dos alimentos descritas por Teixeira (2001).

\footnotetext{
1 Cydectin ${ }^{\circledR}$ NF
} 
As análises para determinação dos valores de MS, PB, FDN, FDA e EE do feno de Tifton 85 utilizado foram realizadas no Laboratório de Nutrição Animal da Escola de Medicina Veterinária da UFBA.

Os ajustes nas quantidades de alimento concentrado fornecidas a cada animal, durante o período do confinamento, foram feitos sempre após as pesagens semanais individuais de todos os animais.

Os cordeiros também foram pesados no dia do nascimento, ao desmame e aos 126 dias, para obtenção dos parâmetros produtivos GPND, GPD, GPC e CAC. Todas as pesagens foram realizadas pela manhã, após jejum de alimento sólido de 13 horas.

Utilizou-se delineamento inteiramente casualizado em esquema fatorial $4 \times 2$, ou seja, quatro idades de desmame (56, 70, 84 e 98 dias de idade) e sexo, com quatro repetições por tratamento, totalizando 32 animais (16 machos e 16 fêmeas).

Os tratamentos foram classificados da seguinte forma:

T1 - machos desmamados aos 56 dias de idade

T2 - fêmeas desmamadas aos 56 dias de idade

T3 - machos desmamados aos 70 dias de idade

T4 - fêmeas desmamadas aos 70 dias de idade

T5 - machos desmamados aos 84 dias de idade

T6 - fêmeas desmamadas aos 84 dias de idade

T7 - machos desmamados aos 98 dias de idade

T8 - fêmeas desmamadas aos 98 dias de idade

Avaliou-se ganho de peso diário do nascimento ao desmame (GPND), ganho de peso diário no confinamento (GPC), ganho de peso diário do nascimento ao abate (GPD) e conversão alimentar do concentrado no confinamento (CAC).

Os resultados foram analisados pelo programa SISVAR (Sistema de Análise de Variância para dados balanceados), desenvolvido por Ferreira (2000), utilizando-se análise de regressão e o seguinte modelo estatístico:

$$
\mathrm{Y}_{\mathrm{ijk}}=\mathrm{m}+\mathrm{i}_{\mathrm{i}}+\mathrm{s}_{\mathrm{j}}+\mathrm{is}_{\mathrm{ij}}+\mathrm{e}_{\mathrm{ij}}
$$

em que $Y_{i j k}$ é o valor da variável observada no cordeiro "k" com idade de desmame i e sexo j; m é a constante inerente ao modelo; $\mathrm{i}_{\mathrm{i}}$, o efeito da idade de desmame, sendo $i=1,2,3,4 ; s_{j}$, o efeito do sexo $j$, sendo $j=1,2$; is ${ }_{\mathrm{ij}}$, o efeito da interação entre a idade de desmame i e o sexo j; $\mathrm{e}_{\mathrm{ij}}$, o erro aleatório associado a cada observação, suposto normalmente distribuído e independente, com média zero e variância $\sigma^{2}$.

\section{Resultados e Discussão}

Constam na Tabela 1 os valores de ganho de peso diário do nascimento ao desmame (GPND).

Não houve efeito da interação $(\mathrm{P}>0,05)$ dos fatores idade de desmame e sexo sobre o GPND (Tabela 1).

O GPND médio observado neste trabalho foi de $127,36 \mathrm{~g}$ (Tabela 1), similar aos valores médios citados por Combellas (1980), de 122,50 g, para algumas raças tropicais, por Matika et al. (2003), de 124 g, para a raça Sabi, e por Godfrey \& Dodson (2003), de 118,8 g, em cordeiros Barbados Blackbelly e Saint Croix White desmamados aos 63 dias de vida.

GPND inferiores foram descritos por Butterworth et al. (1968), de $93 \mathrm{~g}$, em cordeiros Black Headed Persian desmamados aos 84 dias de idade, por Combellas (1981), de 114,3 g, em cordeiros West African suplementados em alimentador restrito, e por Abegaz et al. (2003), de 100 g, em cordeiros Ethiopian Horro desmamados aos 90 dias.

Tabela 1 - Ganho de peso diário do nascimento ao desmame (GPND), em gramas, de acordo com a idade de desmame e o sexo

Table 1 - Daily weight gain (g) from birth to weaning (DWBW), according to weaning age and sex

\begin{tabular}{lccccc}
\hline & \multicolumn{5}{c}{$\begin{array}{c}\text { Idade de desmame (dias) } \\
\text { Weaning age (days) }\end{array}$} \\
\cline { 2 - 4 } Sexo (Sex) & 56 & 70 & 84 & 98 & Média (Mean) \\
\hline Macho (Male) & $117,46^{\mathrm{A}}$ & $142,14^{\mathrm{A}}$ & $130,36^{\mathrm{A}}$ & $119,37^{\mathrm{A}}$ & $127,33^{\mathrm{A}}$ \\
Fêmea (Female) & $123,66^{\mathrm{A}}$ & $133,22^{\mathrm{A}}$ & $129,17^{\mathrm{A}}$ & $123,47^{\mathrm{A}}$ & $127,38^{\mathrm{A}}$ \\
Média (Mean) & 120,56 & 137,68 & 129,76 & 121,42 & 127,36 \\
CV & & & & $11,03 \%$ \\
\hline
\end{tabular}

${ }^{1}$ Médias seguidas de letras maiúsculas diferentes nas colunas, diferem $(P<0,05)$ entre si pelo teste $F$

${ }^{1}$ Means followed by different capital letters in the columns differ $(P<0.05)$ by $F$ test. 


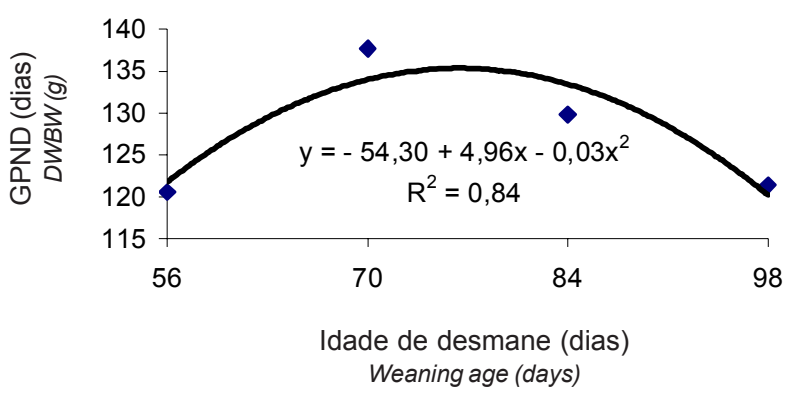

Figura 1 - Efeito da idade de desmame sobre o ganho de peso do nascimento ao desmame (GPND).

Figure 1 - Effect of weaning age on daily weight gain from birth to weaning (DWBW).

Silva et al. (1993) observaram, em cordeiros mestiços Santa Inês provenientes de partos simples desmamados aos 56 e 84 dias de idade, GPND de 158,6 g e $144,4 \mathrm{~g}$, respectivamente, valores superiores aos encontrados neste trabalho.

Variações observadas para valores de GPND podem ser atribuídas a diversos fatores: potencial genético dos cordeiros para ganho de peso (Santos et al., 1999), qualidade da dieta fornecida (Sá \& Otto de Sá, 2002), sistema de produção (Macedo et al. 1999), quantidade de leite produzida pelas ovelhas (Sañudo et al., 1998), contaminação ambiental por helmintos (Haile et al., 2002), número de crias por parto, idade de desmame (Sampaio \& Oliveira, 2002) e época de desmame (Silva et al., 1993).

Verificou-se efeito quadrático $(\mathrm{P}<0,05)$ da idade de desmame sobre o GPND (Figura 1), que atinge seu valor máximo nos animais desmamados com aproximadamente 76 dias de vida. Segundo Oliveira (2002), o ganho de peso dos ovinos é crescente até os 90 dias de idade, fase de maior capacidade de crescimento. $\mathrm{O}$ GPND pode diminuir a partir dos 76 dias de vida, como efeito das infestações helmínticas em ambiente úmido (Boas, 1990), acentuado à medida em que se eleva a idade de desmame dos cordeiros (Haile et al., 2002).

Não houve efeito significativo $(\mathrm{P}>0,05)$ do sexo sobre o GPND (Tabela 1), corroborando os resultados encontrados por Carvalho et al. (1999), em cordeiros mestiços Texel x Ideal desmamados aos 50 dias de idade, e por Guimarães Filho et al. (2000), em cordeiros mestiços Santa Inês desmamados aos 112 dias. Segundo os autores, quanto menor a idade dos ovinos, menor a possibilidade de diferença de desempenho entre os sexos.

Os valores de ganho de peso diário no confinamento (GPC) estão relacionados na Tabela 2.

Houve efeito significativo $(\mathrm{P}<0,05)$ da interação dos fatores idade de desmame e sexo, uma vez que o GPC dos cordeiros desmamados aos 84 dias foi superior aos das cordeiras desmamadas com a mesma idade (Tabela 2).

O GPC médio observado nos ovinos desmamados aos 84 dias, de 178,79 g (Tabela 2), está inserido no intervalo proposto por Barros et al. (1997), de 44 a $267 \mathrm{~g} /$ dia para cordeiros da raça Santa Inês.

Valores de GPC inferiores foram relatados por Camurça et al. (2002), de 98 g, em animais Santa Inês com 26,5 kg de PV inicial, confinados por 42 dias, por Gaili et al. (1992), de 83,3 g/dia a 113,1 g/dia, em cordeiros desmamados com 45, 60, 75 e 90 dias para abate aos nove meses de idade, e por Fernandes et al. (1996), de 156 g, em cordeiros Santa Inês x Crioula desmamados e confinados aos 84 dias de idade recebendo dieta concentrada na proporção de $4 \%$ do PV.

No entanto, GPC superiores aos obtidos neste trabalho foram reportados por Furusho Garcia et al. (2001), de 194,5 g/dia, em ovinos Santa Inês confinados com $15 \mathrm{~kg}$ de PV inicial e alimentados com dieta contendo $80 \%$ de alimento concentrado, formulada

Tabela 2 - Ganho de peso diário no confinamento (GPC), em gramas, de acordo com a idade de desmame e o sexo Table 2 - Daily weight gain in confinement (DWC), in grams, according to weaning age and sex

\begin{tabular}{lccccc} 
& \multicolumn{5}{c}{$\begin{array}{c}\text { Idade de desmame (dias) } \\
\text { Weaning age (days) }\end{array}$} \\
\cline { 2 - 5 } Sexo (Sex) & 56 & 70 & 84 & 98 & Média (Mean) \\
\hline Macho (Male) & $149,29^{\mathrm{A}}$ & $129,91^{\mathrm{A}}$ & $211,31^{\mathrm{A}}$ & $151,79^{\mathrm{A}}$ & 160,57 \\
Fêmea (Female) & $138,57^{\mathrm{A}}$ & $143,30^{\mathrm{A}}$ & $146,28^{\mathrm{B}}$ & $130,36^{\mathrm{A}}$ & 139,63 \\
Média (Average) & 143,93 & 136,61 & 178,79 & 141,07 & 150,10 \\
CV & & & & $19,72 \%$ \\
\hline
\end{tabular}

${ }^{1}$ Médias seguidas de letras maiúsculas diferentes nas colunas, diferem $(P<0,05)$ entre si pelo teste $F$.

${ }^{1}$ Means followed by different capital letters in the columns, differ $(P<0.05)$ by $F$ test. 
para GPC de 300 g/dia, segundo o NRC (1985), e por Barbosa (2002), de 205,61 g/dia, em cordeiros Santa Inês alimentados, do desmame (aos 75 dias) até atingirem $28 \mathrm{~kg}$ de PV, com dieta à base de feno de Buffel grass (Cenchrus cilaris) e alimento concentrado à vontade.

Não foi possível definir uma equação de regressão de primeiro ou de segundo grau que se ajustasse aos dados. A equação cúbica significativa não permite uma interpretação adequada para o parâmetro estudado.

Os dados obtidos para ganho de peso diário do nascimento ao abate (GPD) são apresentados na Tabela 3.

Observou-se efeito significativo $(\mathrm{P}<0,05)$ da interação idade de desmame e sexo sobre o GPD dos animais, em que os indivíduos do sexo masculino desmamados com aproximadamente 84 dias evidenciaram superioridade sobre as cordeiras desmamadas com a mesma idade. Os ovinos desmamados aos 84 dias de idade apresentaram GPD médio de 146,23 g (Tabela 3), valor próximo ao verificado por Barros et al. (1997), de 141,55 g, em cordeiros machos inteiros mestiços Santa Inês, com aproximadamente quatro meses de idade, confinados por 70 dias.

Combellas (1981) relatou GPD de $93 \mathrm{~g}$, em cordeiros West African suplementados em alimentador restrito até o desmame, aos 56 ou 84 dias, e confinados até os 140 dias de idade. De Alba \& Foote (1977) registraram GPD de $107 \mathrm{~g}$, em cordeiros Peliguey com 240 dias de vida, enquanto Combellas (1980) verificou GPD de $115 \mathrm{~g}$ para algumas raças tropicais.

Valores superiores aos alcançados neste trabalho foram obtidos por Carvalho et al. (1999), de $227 \mathrm{~g}$, em cordeiros Texel x Ideal desmamados aos 50 dias e pesados aos 100 dias, e por Muniz et al. (1998), de $189 \mathrm{~g}$, em cordeiros Corriedale e Ideal desmamados aos 60 dias e abatidos aos 100 dias de idade. Carvalho et al.
(1999) e Muniz et al. (1998) confinaram os cordeiros juntamente com as matrizes desde o nascimento e forneceram dietas para atendimento de todos os requisitos nutricionais, tanto das ovelhas lactantes quanto dos cordeiros desmamados.

Sañudo et al. (1998) comentam que dietas com altos níveis de energia, quando fornecidas às matrizes lactantes, elevam as produções de leite e, conseqüentemente, aumentam a ingestão láctea por parte dos cordeiros, promovendo maior PV ao desmame, assim como, quando fornecidas aos cordeiros, proporcionam maiores ganhos de peso após o desmame.

Verificou-se efeito quadrático $(\mathrm{P}<0,05)$ da idade de desmame sobre o GPD, que atingiu valor máximo nos animais desmamados aproximadamente aos 75 dias de idade (Figura 3), corroborando relatos de Vasconcelos et al. (2000), Oliveira et al. (1996) e Castillo et al. (1973), que recomendam as faixas de 70 a 83 dias, de 70 a 84 dias e de 75 a 90 dias, respectivamente.

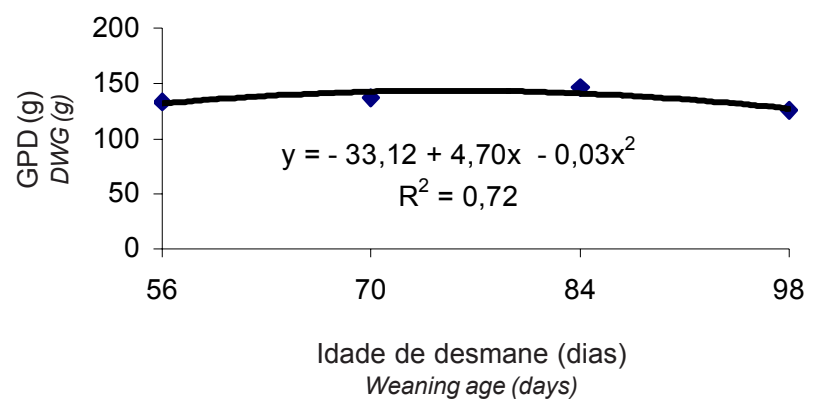

Figura 2 - Efeito da idade de desmame sobre o ganho de peso diário do nascimento ao abate (GPD).

Figure 2 - Effect of weaning age on daily weight gain from birth to slaughter (DWG).

Tabela 3 - Ganho de peso diário do nascimento ao abate (GPD), em gramas, de acordo com a idade de desmame e o sexo

Table 3 - Daily weight gain from birth to slaugther (DWG), in grams, according to weaning age and sex

\begin{tabular}{lccccc}
\hline & \multicolumn{5}{c}{$\begin{array}{c}\text { Idade de desmame (dias) } \\
\text { Weaning age (days) }\end{array}$} \\
\cline { 2 - 5 } Sexo (Sex) & 56 & 70 & 84 & 98 & Média (Mean) \\
\hline Macho (Male) & $135,12^{\mathrm{A}}$ & $136,71^{\mathrm{A}}$ & $157,34^{\mathrm{A}}$ & $125,80^{\mathrm{A}}$ & 138,74 \\
Fêmea (Female) & $131,95^{\mathrm{A}}$ & $137,70^{\mathrm{A}}$ & $135,12^{\mathrm{B}}$ & $125,00^{\mathrm{A}}$ & 132,44 \\
Médias (Mean) & 133,53 & 137,20 & 146,23 & 125,40 & 135,59 \\
CV & & & & & $9,68 \%$ \\
\hline
\end{tabular}

${ }^{1}$ Médias seguidas de letras maiúsculas diferentes nas colunas, diferem $(P<0,05)$ entre si pelo teste $F$.

${ }^{1}$ Means followed by different capital letters in the columns, differ $(P<0.05)$ by $F$ test. 
Houve efeito quadrático da idade de desmame sobre o GPD dos cordeiros (Figura 4), demonstrando que a melhor idade de desmame para animais do sexo masculino é de 76 dias.

Constam na Tabela 4 os valores observados para conversão alimentar do concentrado no confinamento (CAC).

Não houve efeito significativo $(\mathrm{P}>0,05)$ da interação dos fatores idade de desmame e sexo $(\mathrm{P}>0,05)$, nem do sexo sobre a CAC (Tabela 4), possivelmente pelo fato de os animais terem sido abatidos em idade precoce.

A CAC média foi de 2,03:1 (2,03 kg de alimento concentrado $/ \mathrm{kg}$ de ganho de peso vivo), valor menor que o observado por Potkanski et al. (1991), de 1,86:1, em cordeiros Merino Polonês, com GPC's entre 178 e 206 g, desmamados aos 60 dias de idade e alimentados à vontade com feno e concentrado até completarem 120 dias.

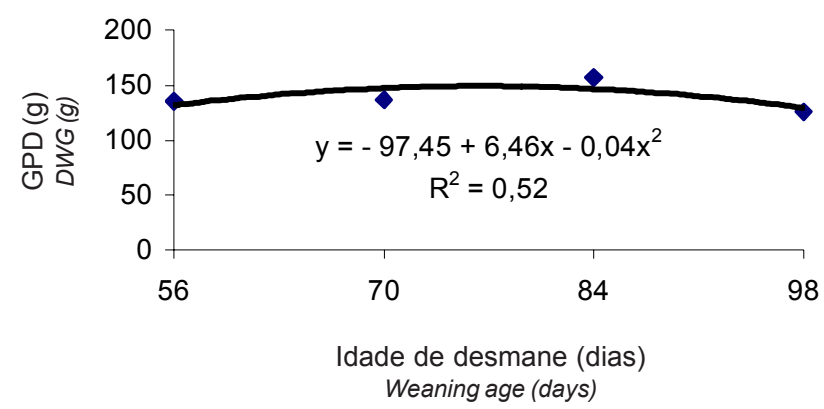

Figura 3 - Efeito da idade de desmame sobre o ganho de peso diário do nascimento ao abate (GPD) em ovinos machos.

Figure 3 - Effect of weaning age on daily weight gain from birth to slaughter (DWG) in male lambs.
A conversão alimentar pode ser afetada pela dieta fornecida aos animais (Rodrigues et al., 1997), pois, segundo Barros et al. (1997), o fornecimento de dietas ricas em alimento concentrado e/ou de maiores níveis de proteína e energia melhoram a eficiência alimentar (Sá \& Otto de Sá, 2002).

Observou-se efeito quadrático $(\mathrm{P}<0,05)$ da idade de desmame sobre a CAC (Figura 5), que atingiu seu nível máximo de eficiência nos animais desmamados aproximadamente aos 72 dias de idade.

Houve efeito quadrático $(\mathrm{P}<0,05)$ sobre a $\mathrm{CAC}$ de cordeiros (Figura 6) e de cordeiras (Figura 7), cujas idades de desmame nas quais este parâmetro assumiu a maior eficiência ocorreu em torno dos 71 e 72 dias, respectivamente. É provável que animais desmamados a partir destas idades já tenham sofrido, de forma mais expressiva, os efeitos deletérios da verminose, reduzindo a eficiência alimentar e, conseqüentemente, piorando a CAC.

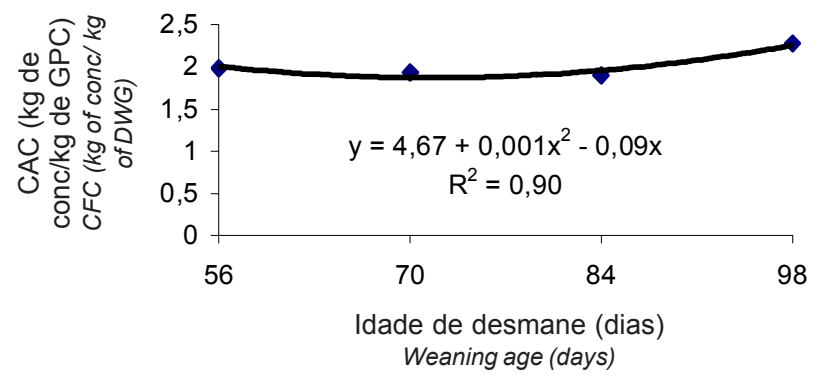

Figura 4 - Efeito da idade de desmame sobre a conversão alimentar do concentrado no confinamento (CAC).

Figure 4 - Effect of weaning age on concentrate food conversion in confinement (CAC).

Tabela 4 - Conversão alimentar do concentrado no confinamento (CAC), de acordo com a idade de desmame e o sexo dos animais

Table 4 - Concentrate feed:gain ratio in confinement (CFC), according to weaning age and sex

\begin{tabular}{|c|c|c|c|c|c|}
\hline \multirow[b]{2}{*}{$\operatorname{Sexo}(\operatorname{Sex})$} & \multicolumn{4}{|c|}{$\begin{array}{c}\text { Idade de desmame (dias) } \\
\text { Weaning age (days) }\end{array}$} & \multirow[b]{2}{*}{ Média (Mean) } \\
\hline & 56 & 70 & 84 & 98 & \\
\hline Macho (Male) & $1,93^{\mathrm{A}}$ & $1,94^{\mathrm{A}}$ & $1,82^{\mathrm{A}}$ & $2,29^{\mathrm{A}}$ & $2,00^{\mathrm{A}}$ \\
\hline Fêmea (Female) & $2,04^{\mathrm{A}}$ & $1,93^{\mathrm{A}}$ & $1,98^{\mathrm{A}}$ & $2,26^{\mathrm{A}}$ & $2,05^{\mathrm{A}}$ \\
\hline Média (Mean) & 1,98 & 1,94 & 1,90 & 2,28 & 2,03 \\
\hline $\mathrm{CV}$ & & & & & $5,98 \%$ \\
\hline
\end{tabular}

${ }^{1}$ Médias seguidas de letras maiúsculas diferentes nas colunas, diferem $(P<0,05)$ entre si pelo teste $F$.

${ }^{1}$ Means followed by different capital letters in the columns, differ $(P<0.05)$ by $F$ test. 


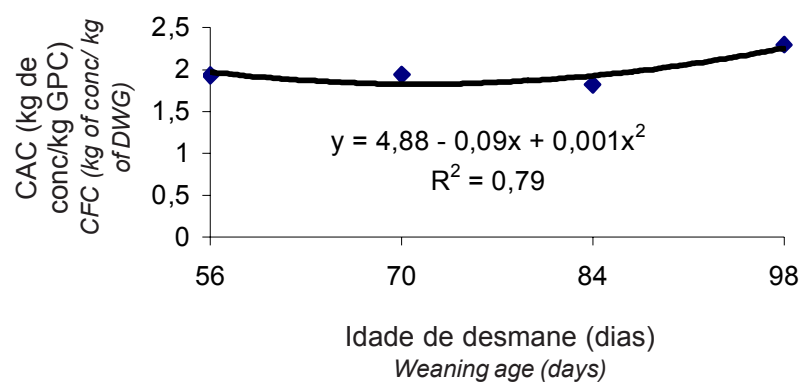

Figura 5 - Efeito da idade de desmame sobre a CAC em ovinos machos.

Figure 5 - Effect of weaning age on concentrate food conversion in confinement (CAC) in male lambs.

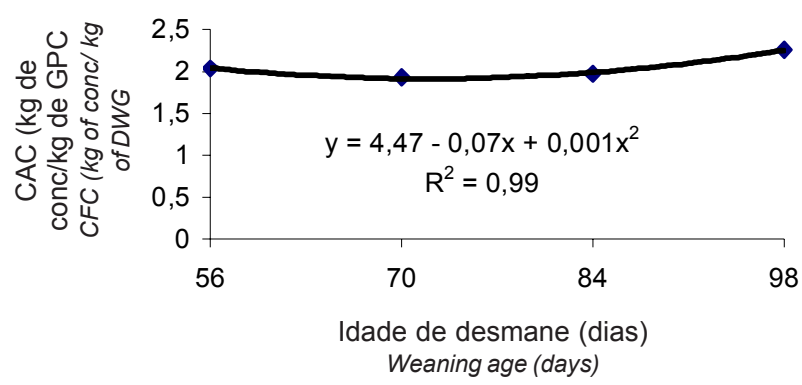

Figura 6 - Efeito da idade de desmame sobre a conversão alimentar do concentrado no confinamento (CAC), em cordeiras.

Figure 6 - Effect of weaning age on concentrate food conversion in confinement (CAC) in female lambs.

\section{Conclusões}

A melhor idade para o desmame de cordeiros deslanados mestiços Santa Inês, para terminação em confinamento, no litoral norte da Bahia, é de 72 dias, uma vez que os animais desmamados com esta idade apresentaram a melhor conversão alimentar em confinamento e os melhores ganhos de peso diário, do nascimento ao desmame e do nascimento ao abate, foram obtidos quando os animais foram desmamados aos 76 e 75 dias, respectivamente.

\section{Literatura Citada}

ABEGAZ, S.; GEMEDA, D.; REGE, J.E.O. et al. Early growth, survival and litter size in Ethiopian Horro sheep. South African Journal of Animal Science, 2000. Disponível em: <http://www.sasas.co.za/Sajas.html $>$. Acesso em: 15 jun. 2003.
BARBOSA, J.A. Sistemas de produção de cordeiros da raça Santa Inês. Cruz das Almas: Universidade Federal da Bahia, 2002. 45p. Dissertação (Mestrado em Zootecnia) - Universidade Federal da Bahia.

BARROS, N.N.; CARVALHO, R.B.; ROSSETI, A.G. Feno de cunhã para acabamento de borregos. In: REUNIÃO ANUAL DA SOCIEDADE BRASILEIRA DE ZOOTECNIA, 34. 1997, Juiz de Fora. Anais... Juiz de Fora: Sociedade Brasileira de Zootecnia, 1997. CD-ROM

BARROS, N.N.; SIMPLÍCIO, A.A.; BARBIERI, M.E. Desempenho de borregos das raças Santa Inês e Somalis Brasileira, em prova de ganho de peso. In: Reunião Anual da Sociedade Brasileira de Zootecnia, 33., 1996, Fortaleza. Anais... Fortaleza: Sociedade Brasileira de Zootecnia, v.1. 1906, p.258.

BARROS, N.N.; SIMPLÍCIO, A.A.; FERNANDES, F.D. Terminação de Borregos em Confinamento no Nordeste do Brasil. Sobral: EMBRAPA-CNPC, 1997. 24p. (Circular Técnica, 12).

BOAS, A.S.V. Instalações e manejo do rebanho ovino. In: PRODUÇÃO DE OVINOS, 1., 1990, Jaboticabal. Anais... Jaboticabal: Fundação de Estudos e Pesquisas em Agronomia, Medicina Veterinária e Zootecnia, 1990. p.27-64.

BUTTERWORTH, M.H.; HOUGHTON, T.R.; MACCARTNEY, J.C. et al. Same observations on the lactation of Blackhead ewes and the growth of lambs: the composition and yield of milk. Journal of Agricultural Science, v.70, p.203-207, 1968.

CAMURÇA, D. A.; NEIVA, J.N.M.; PIMENTEL, J. C. M et al. Desempenho produtivo de ovinos alimentados com dietas à base de feno de gramíneas tropicais. Revista Brasileira de Zootecnia, v.31, n.5, p.2113-2122, 2002.

CARVALHO, S.; PIRES, C.C.; PERES, J.R.R. et al. Desempenho de cordeiros machos inteiros, castrados e fêmeas, alimentados em confinamento. Ciência Rural, v.29, n.1, p.129-133, 1999.

CASTILlO, R.N.; PONCE, H.R.; BERRUECOS, J.M. Caracteristicas de crescimiento del cordero Tabasco I Efecto de la edad y peso al deteste y su influencia sobre la fertilidad de la Madre. Tecnica Pecuaria, p.28-42, 1973.

COMBELLAS, J.B. Growth West African sheep weaned at two different ages. Tropical Animal Production, v.6, n.3, 1981.

COMBELLAS, J. Production and reproduction parameters of tropical sheep breeds in improved production systems. Tropical Animal Production, v.5, n.3, 1980.

DE ALBA, J.; FOOTE, W. Crecimiento de corderos Peliguey en pastoreo. In: REUNION LATINOAMERICANA DE PRODUCCION ANIMAL, 6., 1977, Cuba. Anais... Cuba: 1977.

FERNANDES, F.F.; BARROS, N.N.; ARAUJO, M.R.A. et al. Efeitos de dois planos nutricionais sobre o desempenho de cordeiros $F_{1}$ Santa Inês x Crioula em confinamento. Centro Nacional de Pesquisa de Caprinos, 1996. p.69-72.

FERREIRA, D.F. Análises estatísticas por meio do Sisvar para Windows versão 4.0. In: REUNIÃO ANUAL DA REGIÃO BRASILEIRA DA SOCIEDADE INTERNACIONAL DE BIOMETRIA, 45., 2000, São Carlos. Programa e resumos... São Carlos: Universidade Federal de São Carlos, 2000, p.255-258.

FURUSHO GARCIA, I.F.; BONAGURIO, S. PÉREZ, J.R.O. Desempenho e características de carcaça de cordeiros de diferentes cruzamentos. In: REUNIÃO ANUAL DA SOCIEDADE BRASILEIRA DE ZOOTECNIA, 37., 2000, Viçosa, MG. Anais... Viçosa, MG: Sociedade Brasileira de Zootecnia, 2000. CD-ROM 
FURUSHO GARCIA, I.F.; PÉREZ, J.R.O.; BONAGURIO, S. et al. Características de carcaça de cordeiros Santa Inês e cruzas Santa Inês com Texel, Ile de France e Bergamácia. In: REUNIÃO ANUAL DA SOCIEDADE BRASILEIRA DE ZOOTECNIA, 38., 2001, Piracicaba. Anais... Piracicaba: Sociedade Brasileira de Zootecnia, 2001. p.1142-44.

GAILI, E.S.E. Effect of weaning age on postweaning feedlot performance and carcass characteristics of Najdi lambs. Animal Husbandry Meat, v.27, n.2, p.61, 1992.

GODFREY, R.W.; DODSON, R.E. Effect of supplemental nutrition around lambing on hair sheep ewes and lambs during the dry and wet seasons in the U.S. Virgin Islands. Journal of Animal Science, n.81, p. 587-593, 2003.

GUIMARÃES FILHO, C.; SOARES, J.G.G.; ARAÚJO, G.C.L. Sistemas de Produção de Carnes Caprina e Ovina no Semiárido Nordestino. In: SIMPÓSIO INTERNACIONAL SOBRE CAPRINOS E OVINOS DE CORTE, 1., 2000, João Pessoa. Anais... João Pessoa: Empresa de Pesauisa Agropecuária da Paraíba, 2000. p.21-33.

HAILE, A.; TEMBELY, S.; ANINDO, D.O. et al. Effects of breed and dietary protein supplementation on the responses to gastrointestinal nematode infections in Ethiopian sheep. Small Ruminant Research, v.44, n.3, p.247-261, 2002.

MACEDO, F.A.F.; SIQUEIRA, E.R; MARTINS, E.N. Desempenho de cordeiros Corriedale puros e mestiços, terminados em pastagem e em confinamento. Arquivo Brasileiro de Medicina Veterinária e Zootecnia, v.51, n.6, p.583-587, 1999.

MATIKA, O.; W.Y.K; J.B.V.; ERASMUS, G.J. et al. A description of growth, carcass and reproductive traits of Sabi sheep in Zimbabwe. Small Ruminat Research, v.48, n.2, p.119-126, 2003.

MUNIZ, E.E.; PIRES, C.C.; BORBA, B.A. et al. Crescimento ponderal de cordeiros Corriedale e Ideal alimentados em confinamento. In: REUNIÃO ANUAL DA SOCIEDADE BRASILEIRA DE ZOOTECNIA, 35., 1998, Botucatu. Anais... Botucatu: Gnosis, 1998. CD-ROM. Nutrição de Ruminantes. SIS-031.

NERES, M.A.; GARCIA, C.A.; MONTEIRO, A.L.G. et al. Níveis de feno de alfafa e forma física da ra, ao no desempenho de cordeiros em creep feeding. Revista Brasileira de Zootecnia, v.3, n.30, p.941-947, 2001. Suplemento 1.

NUDELL, D.; HUGHES, H.; FALLER, T. Critical points for profitability in sheep production. North Dakota University Agricultural Research, outono 1998. Disponível em: < http://www.ag.ndsu.nodak.edu/ndagres/fa1198/ ar11198c.htm.> Acesso em: 06 ago. 2001.

OLIVEIRA, G.J.C. Nutrição, Produtividade e Rentabilidade na Caprino-ovinocultura. In: ENCONTRO DE CAPRINOOVINOCULTORES DE CORTE DA BAHIA, 2., 2002, Salvador. Anais... Salvador: Associação Brasileira dos Criadores de Caprinos e Ovinos da Bahia, 2002. 172p. p.1-15.

OLIVEIRA, N.M.; SILVEIRA, V.C.P.; BORBA, M.F.S. Peso corporal de cordeiros e eficiência reprodutiva de ovelhas Corriedale, segundo diferentes idades de desmame em pastagem natural. Revista Brasileira de Agrociência, v.2, n.2, p.113-116, 1996.

POTKANSKI, A.; URBANIAK, M.; KUJAWA, A. Using of rapeseeds in CJ concentrate mixtures for feeding suckling lambs. Roczniki Akademii Poznaniu Zootechnika, n.41, p.85-96, 1991.
RODRIGUES, A.; PÉREZ, J.R.O.; OLIVEIRA, M.V.M. Desempenho de cordeiros Santa Inês e Bergamácia, terminados em confinamento, usando dejetos de suínos como parte da dieta. In: REUNIÃO ANUAL DA SOCIEDADE BRASILEIRA DE ZOOTECNIA, 34., 1997, Fortaleza. Anais... Fortaleza: Sociedade Brasileira de Zootecnia, 1997. CD-ROM

SÁ, J.L.; OTTO de SÁ, C. Carcaças e carnes ovinas de alta qualidade - revisão. Disponível em: < http:// www.crisa.vet.br/publi_2001/carcaca.htm >Acesso em: mar. 2002.

SAMPAIO, E.A.; OLIVEIRA, G.J.C. Determinação da idade de desmame de cordeiros para confinamento, no recôncavo baiano. In: SEMINÁRIO DE PESQUISA DA UNIVERSIDADE FEDERAL DA BAHIA, 30., 2002, Cruz das Almas. Anais... Cruz das Almas: Universidade Federal da Bahia, 2002.

SANTOS, L.E.; CUNHA, E.A.; BUENO, M.S. et al. Desempenho de cordeiros de raças de corte alimentados com dietas contendo níveis crescentes de polpa cítrica em substituição ao milho. In: REUNIÃO ANUAL DA SOCIEDADE BRASILEIRA DE ZOOTECNIA, 36., 1999, Porto Alegre. Anais... Porto Alegre: Gnosis, 1999. CD-ROM. Nutrição de Ruminantes. NUR-053.

SAÑUDO, C.; SIERRA, I.; OLLETA, J. L. Influence of weaning on carcass quality, fatty acid composition and meat quality in intensive lamb production systems. Animal Science, n.66, p.175-187, 1998.

SILVA, F.L.R.; LIMA, F.A.M.; FIGUEIREDO, E.A.P. Desempenho produtivo de ovinos mestiços Santa Inês, no Estado do Ceará. Sobral: Centro Nacional de Pesquisa de Caprinos, 1993. p.36.

SILVA SOBRINHO, A.G. Produção de cordeiros em pastagem. In: SIMPÓSIO MINEIRO DE OVINOCULTURA, 2001, Lavras. Anais... Lavras: Universidade Federal de Lavras, 2001, p.63-97.

SIQUEIRA, E.R. Produção de carne de cordeiro. In: ENCONTRO MINEIRO DE OVINOCULTURA, 1., 2000b, Lavras: Anais... Lavras: Universidade Federal de Lavras, 2000b. p.129-137.

SIQUEIRA, E.R. Sistemas de confinamento de ovinos para corte do sudeste do Brasil. In: SIMPÓSIO INTERNACIONAL SOBRE OVINOS E CAPRINOS DE CORTE, 2000a, João Pessoa. Anais... João Pessoa: Empresa de Pesquisa Agropecuária da Paraíba, 2000a. p.107-117.

TEIXEIRA, A.S. Alimentos e alimentação dos animais. 5.ed. Lavras: Universidade Federal de Lavras/ FAEPE, 2001. 241p.

VASCONCELOS, V.R.; LEITE, E. R.; BARROS, N.N. Terminação de caprinos e ovinos deslanados no Nordeste do Brasil. In: SIMPÓSIO INTERNACIONAL SOBRE OVINOS E CAPRINOS DE CORTE, 2000, João Pessoa. Anais... João Pessoa: Empresa de Pesquisa Agropecuária da Paraíba, 2000. p.97-106. 